\title{
Impacto de la pandemia del COVID-19 sobre la salud estomatológica
}

\author{
Impact of the COVID-19 pandemic on dental health \\ César-Augusto Padilla-Avalos ${ }^{1, a}$, Consuelo Marroquín-Soto ${ }^{1, b}$
}

\section{Sr. Editor:}

La pandemia del COVID-19 ha sido un amplio tema de estudio por el gran impacto global, la alta tasa de contagio y mortalidad, el efecto severo sobre la salud sistémica y sus fatales complicaciones; sin embargo, aún se sigue investigando la enfermedad y su repercusión desde distintos enfoques disciplinarios; dado que el contexto mismo de pandemia, ha desencadenado efectos en la salud estomatológica y psicosocial de la población.

Dadas las recomendaciones de la Organización Mundial de la Salud (OMS), los estados han reforzado protocolos de bioseguridad a la población; instaurando medidas a fin de contener la propagación del virus, incorporando así, nuevos hábitos de vida. A propósito de ello, se ha investigado que el uso cotidiano de las mascarillas, ha influenciado en el hábito de higiene oral, y determinaron que la frecuencia del cepillado dental ha disminuido dado que las personas están menos preocupadas por la higiene bucal, por su sonrisa y estética dental generando altos índices de halitosis (1). Este indicador revela un evidente descuido en la salud oral, haciendo susceptible la aparición de lesiones cariosas $\mathrm{y} / \mathrm{o}$ afecciones periodontales.
La emergencia sanitaria, ha afectado fuertemente las actividades económicas, educativas y profesionales. En efecto, la postergación de los servicios de atención odontológica en el ámbito público y las clínicas universitarias de prácticas en Odontología; han limitado el alcance y el control de las necesidades estomatológicas, hecho adicional al impacto que tiene la pandemia sobre la salud mental de los pacientes.

El deterioro del estado psicoemocional producto de la pandemia se ha relacionado con la presencia de enfermedades orales como: lesiones en la mucosa oral, transtorno temporo mandibular (TTM), bruxismo y enfermedad periodontal. En efecto se evidencia que los factores psicológicos como el estrés y la ansiedad asociada a la pandemia, pueden conducir a la intensificación del dolor orofacial, predisponiendo a un mayor riesgo para desarrollar, empeorar y perpetuar los signos y síntomas del bruxismo y TTM (2-4).

El profesional, así como dirige un tratamiento para el manejo de cada urgencia odontológica, debe asumir un rol social coadyuvante en el apoyo psicológico de sus pacientes y preocuparse por la historia social, 
a fin de encontrar alguna relación predisponente a sufrir bruxismo o TTM. En caso que el paciente sufra de estrés o ansiedad, debe ser derivados con especialistas para recibir soporte psicológico. A nivel estomatológico, se recomienda realizar un análisis completo para identificar signos tempranos en el complejo estomatognático, como dolor/desviación/ ruido articular, desgaste patológico dentario u otro signo que evidencie alteración o para función; para poder intervenir con la terapéutica adecuada $(2,5)$. En consecuencia, la atención odontológica debería ser prioritaria respetando las medidas de bioseguridad, con el fin de redimir las afecciones mencionadas y contribuir con la salud integral de los pacientes.

Conflicto de intereses: Los autores no tienen conflicto de interés con este informe.

Contribuciones de los autores: Todos los autores contribuyeron a este manuscrito.

\section{Correspondencia:}

Cd. Mg. César Augusto Padilla Avalos

Jr. Huamanpoma \#846. San Martín de Porres - Lima

- Perú.

Correo electrónico: cesarpadilla160@gmail.com

\section{REFERENCIAS BIBLIOGRÁFICAS}

1. Pinzan-Vercelino CR, Freitas KM, Girão VM, da Silva DO, Peloso RM, Pinzan A. Does the use of face masks during the COVID-19 pandemic impact on oral hygiene habits, oral conditions, reasons to seek dental care and esthetic concerns? J Clin Exp Dent. 2021; 13(4):e369-e375.

2. Qu X, Zhou X. [Psychological intervention for patients with oral disease during the pandemic period of COVID-19]. Chinese Journal of Stomatology. 2020;55(4):235-240.

3. Emodi-Perlman A, Eli I, Smardz J, et al. Temporomandibular disorders and bruxism outbreak as a possible factor of orofacial pain worsening during the COVID-19 Pandemic - Concomitant Research in Two Countries. J Clin Med. 2020; 9:3250.

4. Almeida-Leite CM, Stuginski-Barbosa J, Rodrigues PC. How psychosocial and economic impacts of COVID-19 pandemic can interfere on bruxism and temporomandibular disorders? J Appl Oral Sci. 2020; 28:e20200263.

5. Dadnam D, Dadnam C, Al-Saffar H. Pandemic bruxism. Br Dent J. 2021; 230(5):271. 